\title{
Magneto-optical Faraday effect in II-VI based semimagnetic semiconductor nanocrystals
}

\author{
Andriy I. Savchuk ${ }^{* 1}$, Volodymyr I. Fediv ${ }^{1}$, Ihor D. Stolyarchuk ${ }^{2}$, Tetyana A. Savchuk ${ }^{1}$, \\ and Alessio Perrone ${ }^{3}$ \\ ${ }^{1}$ Department of Physics of Semiconductors and Nanostructures, Chernivtsi National University, \\ 2 Kotsubinsky str., 58012 Chernivtsi, Ukraine \\ 2 Department of Theoretical Physics, Drogobych Pedagogical University, 3 Stryiska str., 82100 \\ Drogobych, Ukraine \\ 3 Physics Department and INFN, University of Lecce, Via per Arnesano, 73100 Lecce, Italy
}

Received 12 September 2005, revised 21 October 2005, accepted 22 October 2005

Published online 9 March 2006

PACS 78.20.Ls, 78.66.Hf, 78.67.Bf

Different physical and chemical methods were used to prepare II-VI based semimagnetic semiconductor nanocrystals. A study of magneto-optical Faraday effect in the nanocrystals revealed its enhancement as compared with bulk crystals. It is explained in framework of increase in the oscillator strength of exciton transitions and to the difference between these strengths in an external magnetic field. It was observed almost linear dependence of the Faraday rotation as function of magnetic field strength for nanocrystals at liquid helium temperature in contrast to the dependence with saturation in bulk case and changes in process of a transition of the semimagnetic nanocrystals from the paramagnetic state to a spin glass state.

(C) 2006 WILEY-VCH Verlag GmbH \& Co. KGaA, Weinheim

1 Introduction Quantum nanostructures on the base of semimagnetic semiconductors have been intensively studied in recent years. There are several important lines of investigation in this prospective field. One of them is the study of semimagnetic semiconductor quantum dots in the form of nanoparticles or nanocrystals embedded in dielectric matrix. The main feature of the bulk semimagnetic semiconductors type of $\mathrm{II}_{1-\mathrm{x}} \mathrm{Mn}_{\mathrm{x}} \mathrm{VI}$ is the strong s,p-d spin exchange interaction between the band carriers and the magnetic ions which results in a large Zeeman splitting of the valence and conduction band states, a giant Faraday rotation and the formation of magnetic polarons. It is obvious that when one goes from bulk material to zero-dimensional structures the mentioned magneto-optical phenomena should be modified by strong confinement in all three dimensions. From the point of view of applications it should be noted [1] that the spin states of carrier confined in such zero-dimensional structures could serve as a Q-bit for quantum information processing. The other practical approach [2] is the application of semiconductor nanocrystals doped with a paramagnetic ions as connector material between ferromagnetic metal and semiconductor which could significantly increase spin injection in spintronic devices. These new aspects clearly demonstrate that semimagnetic semiconductor quantum dots open up many possibilities in spin electronics by providing a versitale system for controlling electron spins with many tunable parameters. In this report we present results of magneto-optical studies of II-VI based semimagnetic semiconductor nanocrystals prepared by different physical and chemical techniques.

\section{Experimental procedure}

2.1 Sample preparation In case of II-VI based semimagnetic semiconductors a variety of technological methods has been demonstrated for growth of zero-dimensional structures [3-5]. We have previously

\footnotetext{
* Corresponding author: e-mail: savchuk@chnu.cv.ua, Phone: +380 372584 755, Fax: +380 372514986
} 
reported $[6,8]$ on growth by melting of the semiconductor doped fine powder borosilicate glasses, incorporation of $\mathrm{Cd}_{1-\mathrm{x}} \mathrm{Mn}_{\mathrm{x}} \mathrm{Se}, \mathrm{Cd}_{1-\mathrm{x}} \mathrm{Mn}_{\mathrm{x}} \mathrm{Te}$ nanoparticles into zeolite and polymer matrices, and the growth of $\mathrm{Cd}_{1-\mathrm{x}} \mathrm{Mn}_{\mathrm{x}} \mathrm{Te}$ nanocrystals in $\mathrm{SiO}_{2}$ by the laser deposition method.

In addition to the physical methods we have also used chemical techniques for obtained $\mathrm{Cd}_{1-\mathrm{x}} \mathrm{Mn}_{\mathrm{x}} \mathrm{Te}$ and $\mathrm{Cd}_{1-\mathrm{x}} \mathrm{Mn}_{\mathrm{x}} \mathrm{S}$ nanocrystals. Cadmium chloride $\left(\mathrm{CdCl}_{2}\right)$ and manganese chloride tetrahydrate $\left(\mathrm{MnCl}_{2}\right)$ were mixed in the different mole ratio in polyvinyl alcohol (PVA) aqueous solution. Mercaptoethanol $\left(\mathrm{C}_{2} \mathrm{H}_{5} \mathrm{OSH}\right)$ was added to this mixture to obtain stable colloidal solution. This solution then can be dried on a glass plate at $75^{\circ} \mathrm{C}$ to be transformed into a solid film.

For the doping of nanocrystals by magnetic ions several problems should be solved. The main problem is to prevent a rapid agglomeration. To overcome these difficulties a stabilizing agent was added to a liquid solution containing metal salts and halogen compounds. In the study different materials have been used as such an agent to get best result. The size of the obtained nanoparticles was controlled by changes in the mixing rate of reagents, concentration of stabilizer and the temperature regime.

2.2 TEM, optical and magneto-optical measurements Transmission electron microscopy (TEM) was used in order to confirm the microcrystallinity of the grown samples, estimate shape and determine average size of nanoparticles. Optical absorption and Faraday rotation measurements were carried out on setups with grating spectrometer for wavelength $200-2200 \mathrm{~nm}$, a water-cooled electromagnet for producing magnetic field of $5 \mathrm{~T}$, superconductor solenoid for magnetic fields up to $25 \mathrm{~T}$ and a helium cryogenic system for investigations at (4,2-300) K. It was important for Faraday rotation measurements in case of very small semiconductor particles embedded in thin transparent films to provide high enough sensitivity. The Faraday rotation angles $\theta_{\mathrm{F}}$ about $10^{-4} \mathrm{deg}$ were measured using a balanced photodiode bridge [7]. In this scheme the Wollaston prism used as a beam splitter to create two arms of the bridge.

3 Results and discussion The used electron microscope provided maximal resolution of $0.7 \mathrm{~nm}$. In the micrograph (see example in our previous paper [8]) the $\mathrm{Cd}_{1-\mathrm{x}} \mathrm{Mn}_{\mathrm{x}} \mathrm{Te}$ quantum dots appeared as dark spots on light background of transparent matrix $\mathrm{SiO}_{2}$. The distribution of the radii of spherical dots was well approximated by a Gaussian function and the best result concerning narrow size dispersion was achieved for chemically synthesized nanocrystals. Additional confirmation for this gives optical absorption studies. Optical density of colloidal solution containing $\mathrm{Cd}_{1-\mathrm{x}} \mathrm{Mn}_{\mathrm{x}} \mathrm{Te}$ nanoparticles as a function of wavelength is shown in Fig. 1. The shift of the exciton absorption peak at $2.34 \mathrm{eV}$ towards higher energy is caused mainly by confinement effect due to small size of semiconductor particles. When the sample

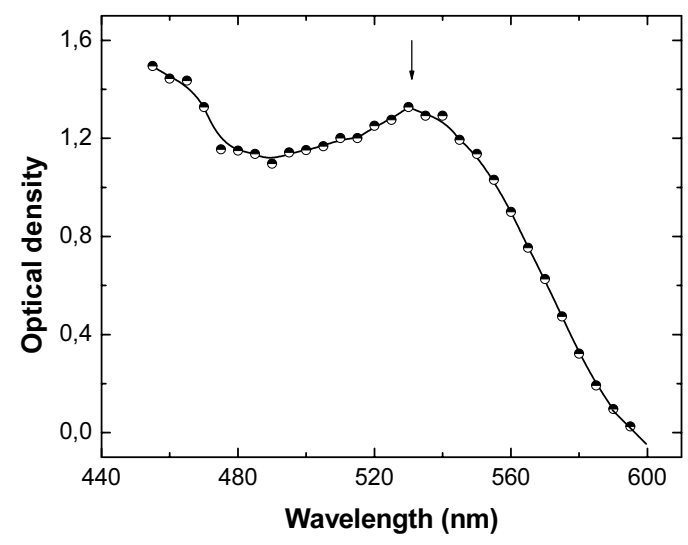

Fig. 1 Optical density of colloidal nanoparticles of $\mathrm{Cd}_{1-\mathrm{x}} \mathrm{Mn}_{\mathrm{x}} \mathrm{Te}$ as a function of wavelength at room temperature.

was cooled to liquid nitrogen temperature the shift of exciton peak towards higher energies but without its significant narrowing could be seen. Obviously, this is result of temperature-independent inhomoge- 
neous broadening which is arisen from the finite size distribution. It was difficult to estimate exact content $\mathrm{x}$ of $\mathrm{Mn}$ in $\mathrm{Cd}_{1-\mathrm{x}} \mathrm{Mn}_{\mathrm{x}}$ Te nanoparticles, but $\mathrm{x}$ not exceed 0.01 .

Nanocrystals with much more content of Mn have been prepared by the laser deposition technique. The broadened absorption edge and absence of excitonic structure are due to not only larger lattice disordering in semiconductor solids solutions with increased value of $\mathrm{x}$ but also worse distribution of the radii quantum dots for this technological method. By thermal annealing of the $\mathrm{Cd}_{1-\mathrm{x}} \mathrm{Mn}_{\mathrm{x}} \mathrm{Te}$ nanocrystals one can reach increase of the size of the nanocrystallites but can not improve their size distribution.

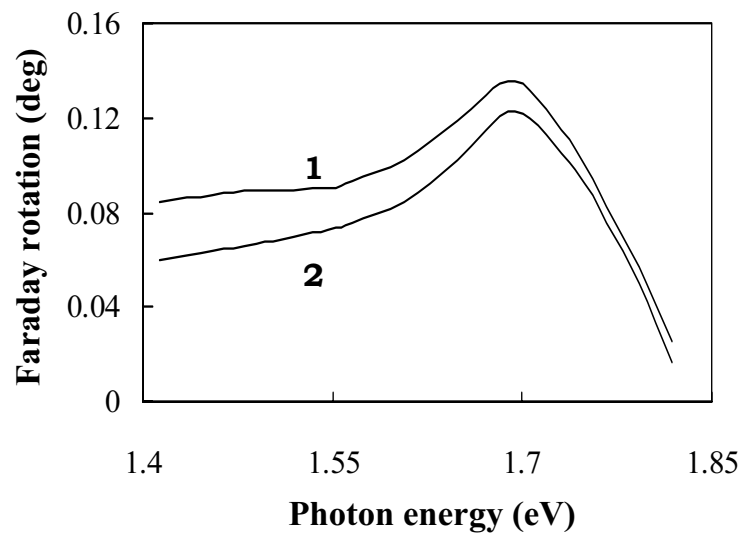

Fig. 2 Spectral dependence of the Faraday rotation for nanocrystals of $\mathrm{Cd}_{0.75} \mathrm{Mn}_{0.25} \mathrm{Te}$ (curve 1) and nanocrystals in $\mathrm{SiO}_{2}$ matrices (curve 2) at room temperature.

The Faraday rotation experiments proved to be very informative on bulk semimagnetic semiconductor crystals [9], in particular because it is related directly to the exchange interaction between the localized magnetic moments of $\mathrm{Mn}^{2+}$ ions and the band-carrier spins. Thus, the Faraday effect can be applied for revealing of features in these processes as a result of lowering of the dimensionality of the semimagnetic semiconductor nanocrystals. Spectral dependence of the Faraday rotation for nanocrystals of $\mathrm{Cd}_{1-\mathrm{x}} \mathrm{Mn}_{\mathrm{x}} \mathrm{Te}$ $(x=0.25)$ grown by the laser deposition technique is shown in Fig. 2. Main features for this sample arenegative sign of the angle of rotation like for bulk crystal and additional enhancement of the Faraday rotation. By using evaluating approach[4] the Verdet constant $\left(\mathrm{V}=\theta_{\mathrm{F}} / \mathrm{Hd}\right)$ of the nanocrystals themselves $\mathrm{V}=0.3 \mathrm{deg} / \mathrm{Oe} \mathrm{cm}$ at $\mathrm{h} v=1.45 \mathrm{eV}$, which is an order of magnitude higher than $\mathrm{V}=0.027 \mathrm{deg} / \mathrm{Oe} \mathrm{cm}$ for bulk $\mathrm{Cd}_{0.75} \mathrm{Mn}_{0.25} \mathrm{Te}$ crystal. This enhancement can be assignment to an increase in the oscillator strength of excitons with left and right circular polarizations and their difference in an external magnetic field.

A study of the Faraday effect in nanocrystals at helium temperatures revealed two additional features. Measurements in strong pulsed magnetic fields of intensity up to $20 \mathrm{~T}$ showed (Fig. 3) that

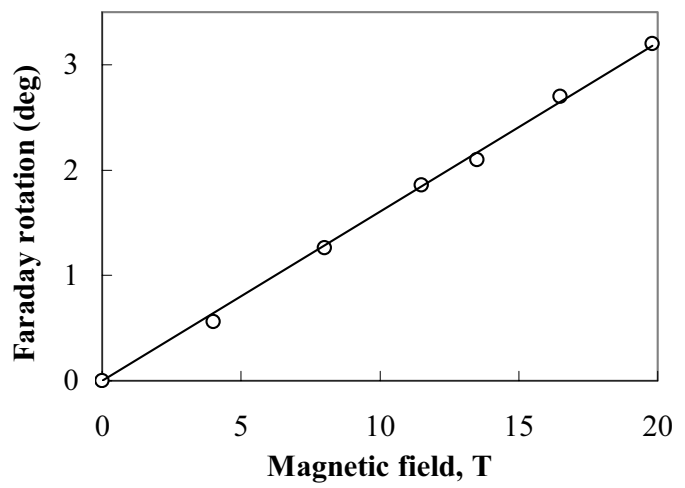

Fig. 3 Magnetic field dependence of the Faraday rotation of $\mathrm{Cd}_{1-\mathrm{x}} \mathrm{Mn}_{\mathrm{x}} \mathrm{Te}$ nanocrystals at $\mathrm{T}=5 \mathrm{~K}$. 
the Faraday rotation angle of the nanocrystals varied linearly in this range, in contrast to the magnetic field dependence showing saturation in high fields, typical for bulk semimagnetic materials.

Another feature was revealed in a study, by means of the Faraday effect, of a transition of this semimagnetic material from paramagnetic state to a spin glass state. At a fixed wavelength we determined the temperature dependence of the Faraday rotation angle in the range $4.2 \mathrm{~K}-30 \mathrm{~K}$ (Fig. 4). The procedure included initial cooling from room temperature to $\mathrm{T}_{\min }=4.2 \mathrm{~K}$ in the absence of a magnetic field, a subsequent application of the field at this temperature, and measurement of $\Theta(T)$ during gradual increase in temperature to $T_{\max }=30 \mathrm{~K}$. In the second cycle of measurements the angle of rotation was recorded during cooling from $T_{\max }$ to $T_{\text {min }}$.

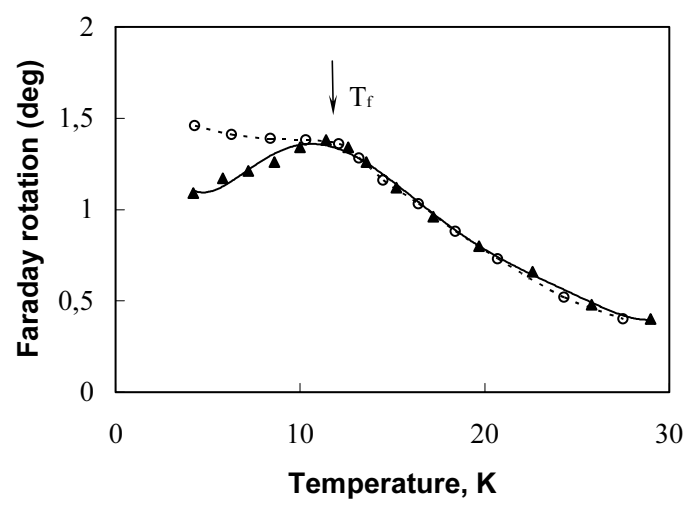

Fig. 4 The angle of the Faraday rotation of $\mathrm{Cd}_{0.75} \mathrm{Mn}_{0.25}$ Te nanocrystals as a function of temperature, when temperature was lowered $(O)$ or increased $(\boldsymbol{\Lambda})$ in the region of the paramagnet-spin-glass transition in magnetic field $\mathrm{H}=$ $0.5 \mathrm{~T}$ at wavelength $\lambda=770 \mathrm{~nm}$.

We thus determined the dependence $\Theta(\mathrm{T})$ typical of the paramagnetic - spin glass transition. A characteristic feature was a considerable shift of the transition temperature $T_{\mathrm{f}}=11 \mathrm{~K}-12 \mathrm{~K}$ for nanocrystals compared with that for bulk semimagnetic semiconductors, equal to $5 \mathrm{~K}-6 \mathrm{~K}$. A similar increase in $\mathrm{T}_{\mathrm{f}}$ on reduction in the nanoparticle size was observed earlier for the magnetic susceptibility of $\mathrm{Cd}_{1-\mathrm{x}} \mathrm{Mn}_{\mathrm{x}} \mathrm{S}$ [10]

Acknowledgements This work has been supported in part by grant No. M/128-2004 from Ministry of Education and Science of Ukraine.

\section{References}

[1] D. Loss and D.P. DiVincenco, Phys. Rev. A 57, 120 (1998).

[2] A.L. Efros, E.I. Rashba, and M. Rosen, Phys. Rev. Lett. 87, 206601 (2001).

[3] K. Yanata, K. Suzuki, and Y. Oka, J. Appl. Phys. 73, 4595 (1993).

[4] H. Nasu, M. Hayashi, J. Matsuoka, K. Kamiya, K. Tanaka, and K. Hirao, Jpn. J. Appl. Phys. 34, L440 (1995).

[5] N. Takahashi, K. Takabayashi, E. Shirado, I. Souma, J.X. Shen, and Y. Oka, J. Cryst. Growth 214/215, 183 (2000).

[6] A.I. Savchuk, I.D. Stolyarchuk, S.V. Medynskiy, A. Perrone, and P.I. Nikitin, Thin Solid Films 336, 176 (1998).

[7] S.A. Crooker, D.D. Awschalom, J.J. Baumberg, F. Flack, and N. Samarth, Phys. Rev. B 56, 7574 (1997).

[8] A.I. Savchuk, I.D. Stolyarchuk, S.V. Medynskiy, V.I. Fediv, Ye.O. Kandyba, A. Perrone, M.L. DeGiorgi, and P.I. Nikitin, Mater. Sci. Eng. C 15, 179 (2001).

[9] J. K. Furdyna, J. Appl. Phys. 64, R29 (1988).

[10] R.J Bandaranayake, M. Smith, J.Y. Lin, H.X. Jiang, and C.M. Sorensen, IEEE Trans. Magn. 30, 493 (1994). 